\title{
Intramuscular Tenosynovial Giant Cell Tumor, Diffuse-Type
}

\author{
Yoo Jin Lee · Youngjin Kang · Jiyoon Jung · Seojin Kim · Chul Hwan Kim \\ Department of Pathology, Korea University Anam Hospital, Seoul, Korea
}

Tenosynovial giant cell tumors (TSGCT), a group of tumors that originate in tendon sheaths, joints, bursae, or adjacent soft tissue, were first described in 1941 by Jaffe et al. ${ }^{1}$ Diffuse-type TSGCT are known to be located in the periarticular soft tissue, while pure intramuscular tumors are rare. This case describes a diffuse-type TSGCT located in the hamstring muscle, which was determined to be a pure intramuscular type.

\section{CASE REPORT}

A healthy 52-year-old Korean woman presented with a 1-month history of right thigh pain and numbness with a palpable mass in the thigh. A T2-weighted magnetic resonance image showed a $7 \mathrm{~cm} \times 5 \mathrm{~cm}$-sized heterogeneous mass with distinct demarcation in the right hamstring muscle (Fig. 1A) that was suggestive of soft tissue sarcoma or nodular fasciitis. The patient was relatively healthy and had no history of previous surgery.

The patient underwent an operation to enucleate the entire mass, and a well-encapsulated yellowish round mass was dissected and removed. Grossly, the resected specimen was a $7 \mathrm{~cm} \times 5$ $\mathrm{cm} \times 2 \mathrm{~cm}$-sized mass and weighed $45 \mathrm{~g}$. The cut surface showed a variegated brown- to yellow-colored appearance with dark reddish punctuated areas and focal myxoid portions (Fig. 1B). Upon microscopic examination, the tumor revealed a villonodular pattern with polygonal mononuclear cells, foamy macrophages and multinucleated giant cells (Fig. 2A, B). Differential

\section{Corresponding Author}

Chul Hwan Kim, MD

Department of Pathology, Korea University Anam Hospital, 73 Inchon-ro, Seongbuk-gu, Seoul 02841, Korea

Tel: +82-2-920-5595, Fax: +82-2-920-6576, E-mail: chkap@korea.ac.kr

Received: September 17, 2015 Revised: October 13, 2015

Accepted: November 15, 2015 diagnosis included tenosynovial giant cell tumor, giant cell tumor of soft tissue, fibromas of the tendon sheath, epithelioid sarcoma, synovial sarcoma, and granulomatous lesions such as tendinous xanthoma. The immunohistochemical stains for CD68, S-100, desmin, epithelial membrane antigen (EMA), and CD34 were performed. The tumor cells showed granular cytoplasmic positivity for CD68 (Fig. 2C) and S-100 but were negative for desmin, EMA, and CD34. TSGCT was suggested based on histopathologic and immunohistochemical findings. A cytogenetic study was performed to confirm the diagnosis.

A nested polymerase chain reaction was performed, using the first and second round primer sets from a previous report (Fig. 2D). ${ }^{2}$ When using the primer set COL6A3-2529F/CSF1-1752R in the first round and COL6A3-2588F/CSF1-1698R (exon 8) in the second round (lane 2), a 1.4-kbp fragment was amplified. Therefore, the cytogenetic result of this case indicated the fusion of colony stimulating factor-1 (CSF1) and collagen type VI alpha-3 (COL6A3), and the tumor was diagnosed as an intramuscular TSGCT diffuse type. This report was approved by the Institutional Review Board of Korea University Anam Hospital (AN15199-001).

\section{DISCUSSION}

TSGCT can be subtyped into diffuse and localized types, and intra-articular and extra-articular types, according to the growth pattern and location. This classification suggests different clinical behaviors. ${ }^{3}$ Diffuse-type TSGCT presents as an extra-articular type in about $5 \%$ to $15 \%$ of cases. ${ }^{4}$ It is a slow-growing lesion with a favorable prognosis, usually treated with complete excision. The tumor can be locally aggressive, even if benign in nature, and $33 \%$ to $50 \%$ can recur, although metastasis to other organs is rare after multiple recurrences. ${ }^{4,5}$

It is usually identified in periarticular tissue and can present 
as extra-articular extension of a primary intra-articular process. It can also reside completely outside of the joint, bursa or tendon sheath. Pure intramuscular type of the tumor has only been reported a few times. A previous study ${ }^{4}$ showed only eight cases of diffuse-type TSGCT that had a clear origin in tendinous or synovial tissue. Six cases were located in a predominantly sub-
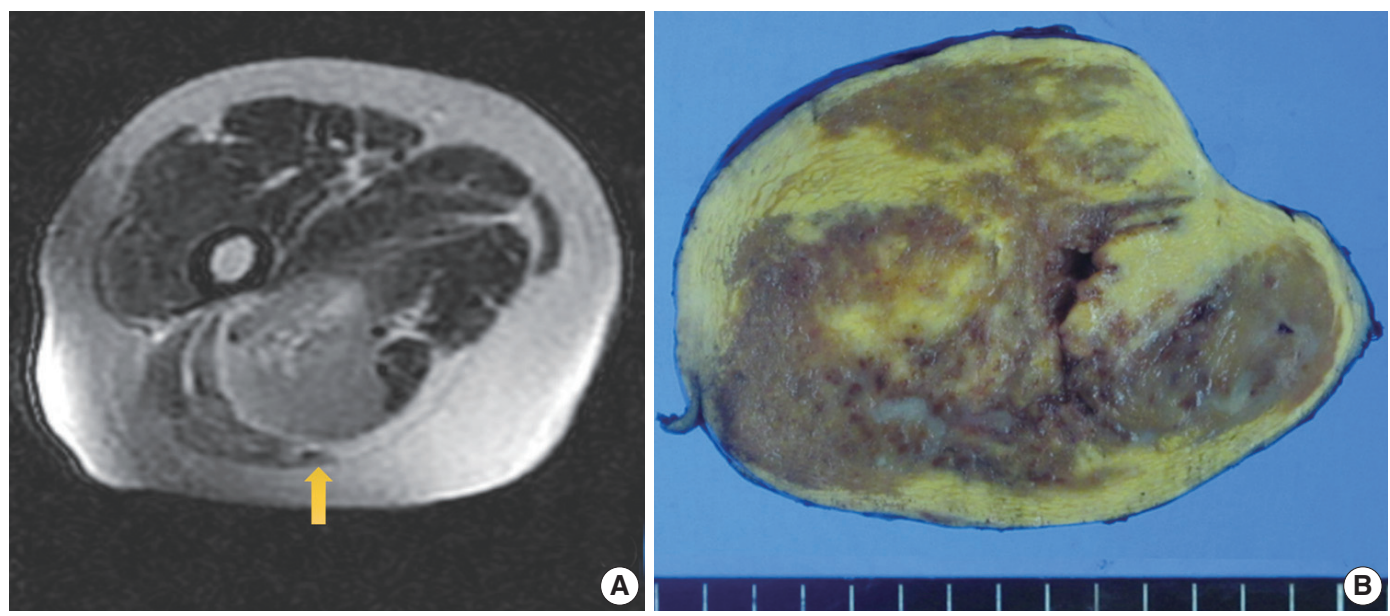

Fig. 1. (A) T2-weighted magnetic resonance image showing a well-marginated mass in the muscle of the right thigh (arrow). (B) The cut surface of the resected specimen shows a variegated brown to yellow colored mass with multifocal dark reddish punctuate areas and focal myxoid portions.
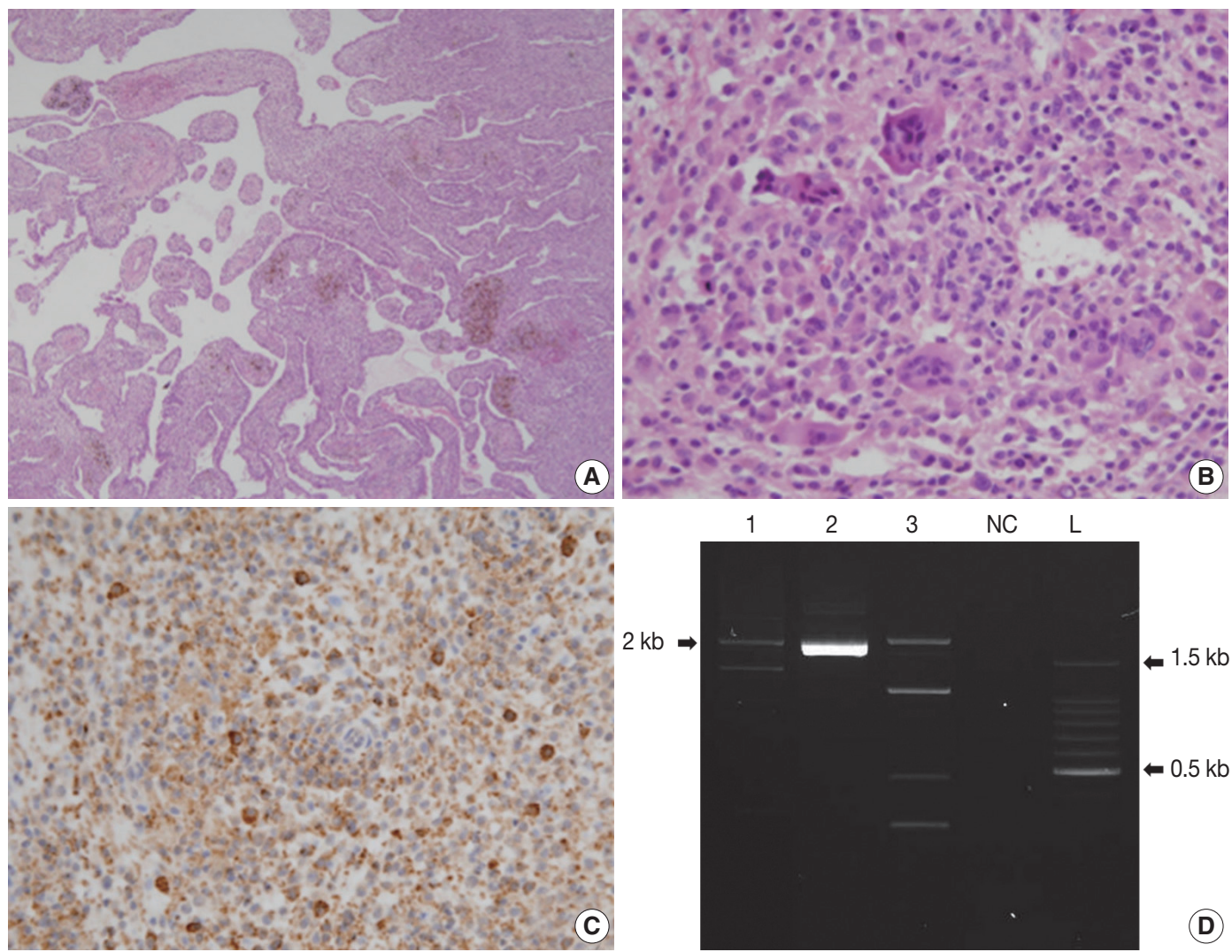

Fig. 2. (A) Hematoxylin-eosin stained slide of a diffuse-type tenosynovial giant cell tumor shows sheets of small histiocytes with a villonodular pattern and hemosiderin pigments. (B) Variable proportions of polygonal mononuclear cells and multinucleated giant cell are seen. (C) The tumor cells are positive for CD68 immunohistochemical staining. (D) Nested polymerase chain reaction to detect the COL6A3-CSF1 fusion transcript (lane 1 and lane 2) and CSF1 wild-type transcripts (lane 3). COL6A3, collagen type VI alpha-3; CSF1, colony stimulating factor-1; $\mathrm{NC}$, negative control; L, ladder. 
cutaneous area, and five cases were entirely intramuscular. The intramuscular type tumor can affect any muscle, but most were located in the lower extremities, including thigh, buttock, and lower leg, ${ }^{4}$ which was similar to our case, while one case from another report ${ }^{6}$ originated in an upper extremity, specifically the deltoid muscle.

This tumor was once regarded as a non-neoplastic condition, but their potential for recurrence and metastasis suggested the possibilities of neoplastic nature. ${ }^{5,7}$ Furthermore, the cytogenetic studies identified clonal abnormalities, which suggested that this tumor was indeed a neoplasm..$^{5}$ An additional study ${ }^{2}$ found a high expression of CSF1, localized to the 1p13q breakpoint, which is a hematopoietic growth factor that involves the proliferation and differentiation of macrophages and monocytes. CSF1 is often fused with COL6A3 on 2q35, which is thought to have a major oncogenic role in TSGCT. Due to the overexpression of CSF1, macrophages proliferate and become the main component of TSGCT. However, about 39\% do not have CSF1 translocation, so the other alternative mechanism may affect CSF1 upregulation.

This case was an intramuscular soft tissue tumor without any connections to joints, tendons, or bursa and presented as a pure intramuscular type. The histologic features showed a villonodular pattern composed of histiocytes, foamy macrophages, multinucleated giant cells, and hemosiderin deposits. On cytogenetic study, this neoplasm was found to have clonal abnormalities, which are evidence for diffuse-type TSGCT. One report ${ }^{6}$ suggested that the recurrence rate of intramuscular-type is lower than that of other types, as complete excision is easier for intramuscular masses than intra-articular lesions. However, the biologic behavior of intramuscular type tumors remains unclear due to the small number of cases, which necessitates further studies to determine the prognostic significance of intramuscular lesions.

\section{Conflicts of Interest}

No potential conflict of interest relevant to this article was reported.

\section{REFERENCES}

1. Jaffe HL, Lichtenstein L, Sutro CJ. Pigmented villonodular synovitis, bursitis and tenosynovitis. Arch Pathol 1941; 31: 731-65.

2. Möller E, Mandahl N, Mertens F, Panagopoulos I. Molecular identification of COL6A3-CSF1 fusion transcripts in tenosynovial giant cell tumors. Genes Chromosomes Cancer 2008; 47: 21-5.

3. Ushijima M, Hashimoto H, Tsuneyoshi M, Enjoji M. Giant cell tumor of the tendon sheath (nodular tenosynovitis). A study of 207 cases to compare the large joint group with the common digit group. Cancer 1986; 57: 875-84.

4. Somerhausen NS, Fletcher CD. Diffuse-type giant cell tumor: clinicopathologic and immunohistochemical analysis of 50 cases with extraarticular disease. Am J Surg Pathol 2000; 24: 479-92.

5. Lucas DR. Tenosynovial giant cell tumor: case report and review. Arch Pathol Lab Med 2012; 136: 901-6.

6. Yun SJ, Hwang SY, Jin W, Lim SJ, Park SY. Intramuscular diffusetype tenosynovial giant cell tumor of the deltoid muscle in a child. Skeletal Radiol 2014; 43: 1179-83.

7. Sanghvi DA, Purandare NC, Jambhekar NA, Agarwal MG, Agarwal A. Diffuse-type giant cell tumor of the subcutaneous thigh. Skeletal Radiol 2007; 36: 327-30. 